\title{
Use-related risk analysis for medical devices based on improved FMEA
}

\author{
Long Liu ${ }^{\mathrm{a}}$, Shuai $\mathrm{Ma}^{\mathrm{b}, *}$, ZhuWang ${ }^{\mathrm{b}}$ and Ping $\mathrm{Li}^{\mathrm{c}, *}$ \\ ${ }^{a}$ College of Design \& Innovation, Tongji University, Room 102, Building B, 1623 Cailun Road, Shanghai, China, \\ 201203 \\ b Institute of Industry Engineering, Tongji University, Room 102, Building B, 1623 Cailun Road, Shanghai, China, \\ 201203 \\ ${ }^{\mathrm{c}}$ Shanghai East Hospital, 150 Jimo Road, Shanghai, China, 200120
}

\begin{abstract}
In order to effectively analyze and control use-related risk of medical devices, quantitative methodologies must be applied. Failure Mode and Effects Analysis (FMEA) is a proactive technique for error detection and risk reduction. In this article, an improved FMEA based on Fuzzy Mathematics and Grey Relational Theory is developed to better carry out userelated risk analysis for medical devices. As an example, the analysis process using this improved FMEA method for a certain medical device (C-arm X-ray machine) is described.
\end{abstract}

Keywords: Use-related risk, medical device, FMEA

\section{Introduction}

The development of medical devices provides powerful tools for diagnosis and treatment of diseases, but also brings terrible medical risk. While the medical diagnosis and device technologies are improved continuously, more and more device-userelated clinical risks are attracting attention[1]. According to a study released by HealthGrades of U.S., among the 2,500,000 accident death of patients from 2000 to $2002,575,000$ of them result from preventable medial errors[2]. It is clear that in order to ensure medical devices provide services as expected, the use-related risk must be analyzed and controlled through effective measures.

\section{Failure mode and effect analysis}

The FMEA process is a "systematic method of identifying and preventing product and process problems before they occur". FMEA focuses on identifying and removing defects, enhancing safety, and increasing customer satisfaction[3]. It can be applied in the design of medical processes in order to prevent errors, accidents and adverse reactions. Examples of field of application are the design of the process of treatment and therapy administration.

Some commonly used terms related to FMEA are specified as follows[4]:

Severity (S): the consequences of a failure mode. Severity considers the worst potential consequence of a failure, determined by the degree of injury, property damage, or system damage that could ultimately occur.

Occurrence $(\boldsymbol{O})$ : the probability of occurrence of a failure mode.

Detection (D): the level that potential causes of a failure mode can be inspected out.

$\boldsymbol{F M E A}$ is a procedure in product development and operations management for analysis of potential failure modes within a system for classification by the severity and likelihood of the failures.

The implementation steps of FMEA are operationally defined as[5]: a) choosing a process to be studied; b) assembling a multidisciplinary team; c) collecting and organizing information on the process studied; d) conducting a hazard analysis; e) develop-

\footnotetext{
*Shuai Ma. E-mail: mashuai111@gmail.com.

*Ping Li. E-mail: mashuai87111@126.com
} 
ing and implementing actions and outcomes measures.

\section{FMEA applied in use-related risk analysis for medical devices}

As an analytical methodology, FMEA can be very effective in improving process management and project management, but when it comes into medical sector, the particularities of this industry[6] (E.g.: the uncertainty of health care service standard causes difficulties in identifying failure mode; experts' subjective analysis determines the lack of objectivity of acquired data) make its application limited.

In this article, an improved FMEA method based on Fuzzy Mathematics and Grey Relational Analysis is developed to better carry out use-related risk analysis for medical devices. The operational process is shown in Fout! Verwijzingsbron niet gevonden..

As an example, the main analysis processes based on FMEA on the use-related risk of C-arm X-ray machine (see Fout! Verwijzingsbron niet gevonden.) - a specified medical device - are described.

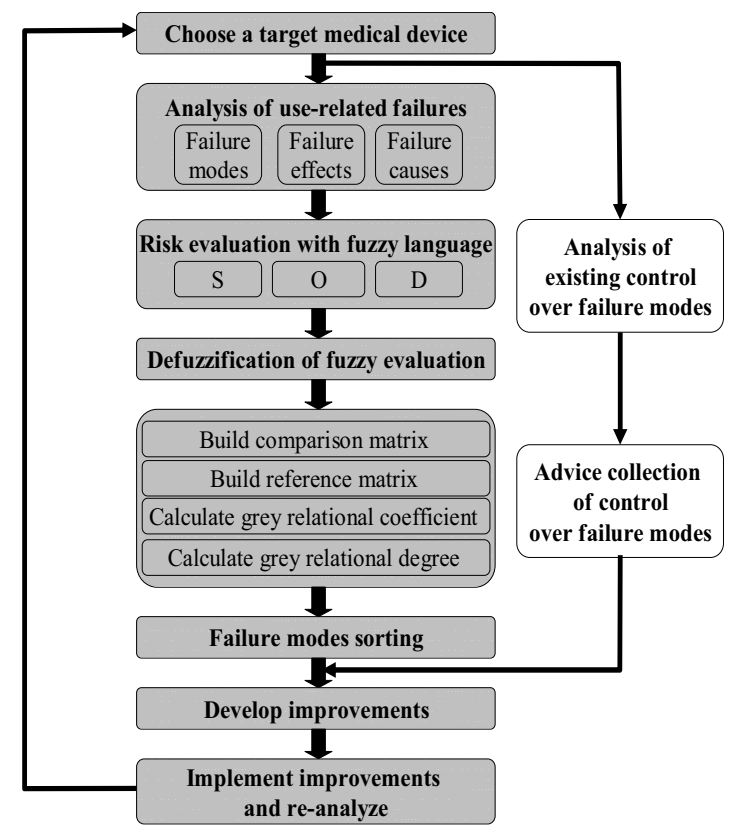

Figure 1 Improved FMEA process based on Fuzzy Mathematics and Grey Relational Analysis

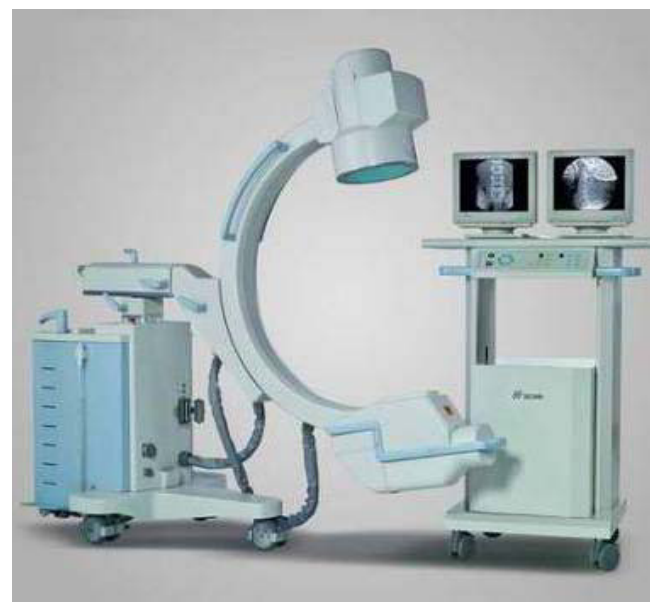

Figure $2 \mathrm{C}$-arm X-ray machine

\subsection{Analysis of use-related failures}

After deep investigation into the use features and processes of $\mathrm{C}$-arm machine, a use-related failure mode framework is developed (see Table 1). $F_{i}$ is a certain failure mode; $F_{i j}$ is the failure cause $j$ in failure mode $\mathrm{F}_{\mathrm{i}}$.

When implementing the investigation, the existing risk control information is requested to provide.

\subsection{Risk evaluation with fuzzy language}

\subsubsection{Define fuzzy language set}

Fuzzy set theory applies fuzzy numbers to describe subjective and uncertain information quantitatively[7]. The fuzzy language set in FMEA is shown in Fout! Verwijzingsbron niet gevonden.

\subsubsection{Determine the membership function}

Considering the applicability and easiness, here triangle membership function is used to give examples. Triangle fuzzy number is generally expressed as $\mathrm{A}=(\mathrm{a}, \mathrm{b}, \mathrm{c})$. Its membership function is as Eq. (1)

$$
\mu_{A}(x)= \begin{cases}\frac{x-a}{b-a}, & a \leq x \leq b \\ \frac{c-x}{c-b}, & b<x \leq c \\ 0, & \text { otherelse }\end{cases}
$$


Table 1

Use-related failures analysis of C-arm X-ray machine

\begin{tabular}{|c|c|c|}
\hline Failure Mode & Failure Effect & Failure Causes \\
\hline \multirow{2}{*}{$\begin{array}{l}F_{1} \text { Ineffective implementation of device } \\
\text { use rules }\end{array}$} & \multirow[t]{2}{*}{ Poor use; device failure; surgery risk } & $\mathrm{F}_{11}$ Improper device use specifications \\
\hline & & $F_{12}$ User changes use steps/manner without authorization \\
\hline \multirow{3}{*}{$\begin{array}{l}\mathrm{F}_{2} \text { Ineffective implementation of device } \\
\text { overhaul system }\end{array}$} & \multirow{3}{*}{$\begin{array}{l}\text { Unable to pre-identify and eliminate } \\
\text { device failure }\end{array}$} & $\mathrm{F}_{21}$ Improper device overhaul system \\
\hline & & $\mathrm{F}_{22}$ Maintenance man changes maintenanc \\
\hline & & $\mathrm{F}_{23}$ Maintenance personnel lack skills/awareness \\
\hline \multirow{2}{*}{$\mathrm{F}_{3}$ Device fatigue /overuse } & \multirow{2}{*}{$\begin{array}{l}\text { Cause device failure; increase patient } \\
\text { danger }\end{array}$} & $\mathrm{F}_{31}$ Lack of devices \\
\hline & & $\mathrm{F}_{32}$ Conflicts of device use arrangement \\
\hline \multirow{3}{*}{$\begin{array}{l}\mathrm{F}_{4} \text { Unable to master right/practical opera- } \\
\text { tion procedure }\end{array}$} & \multirow{3}{*}{$\begin{array}{l}\text { Use error; cause surgery risk acci- } \\
\text { dents }\end{array}$} & $\mathrm{F}_{41}$ Training received but exam failed \\
\hline & & $\begin{array}{l}\mathrm{F}_{42} \text { Training received, exam passed, but right operations } \\
\text { not mastered }\end{array}$ \\
\hline & & $\mathrm{F}_{43}$ Improper training/exam content/manner \\
\hline \multirow{4}{*}{$\mathrm{F}_{5}$ Inaccurate operation orientation } & \multirow{4}{*}{$\begin{array}{l}\text { Device damage; surgery/patient } \\
\text { safety affected }\end{array}$} & $\mathrm{F}_{51}$ Operation qualified, but lack training \\
\hline & & $\mathrm{F}_{52}$ Operation qualified, but use illegally \\
\hline & & $\mathrm{F}_{53}$ Poor device interface design, causes use \\
\hline & & \\
\hline \multirow{3}{*}{$\begin{array}{l}\mathrm{F}_{6} \text { Posture moved to suit device use dur- } \\
\text { ing surgery }\end{array}$} & \multirow{3}{*}{$\begin{array}{l}\text { Extend surgery time; increase pic- } \\
\text { ture-taken times; operators and pa- } \\
\text { tient absorb more irradiation }\end{array}$} & $\mathrm{F}_{61}$ Lack o \\
\hline & & $\begin{array}{l}\mathrm{F}_{62} \text { Poor understanding of purpose/requirement of taking } \\
\text { pictures }\end{array}$ \\
\hline & & $\mathrm{F}_{63}$ Lack of communication with attending physician \\
\hline \multirow{4}{*}{$\mathrm{F}_{7}$ Inadequate radiation protection } & \multirow{4}{*}{$\begin{array}{l}\text { Body disease caused by radiation; } \\
\text { health threats to use/patient }\end{array}$} & $\mathrm{F}_{71}$ Limited protective condition \\
\hline & & $\mathrm{F}_{72}$ Improper existing protection system \\
\hline & & $\mathrm{F}_{73}$ Lack of protection training \\
\hline & & $\mathrm{F}_{74}$ Weak awareness of self-pro \\
\hline \multirow{2}{*}{$\begin{array}{l}F_{8} \text { Disqualification of surgery environ- } \\
\text { ment }\end{array}$} & \multirow{2}{*}{$\begin{array}{l}\text { Operations/device use affected; } \\
\text { cause surgery infection }\end{array}$} & $\mathrm{F}_{81}$ Users' operation affected by noise \\
\hline & & $\mathrm{F}$ \\
\hline
\end{tabular}

Table 2

Definition of evaluation languages

\begin{tabular}{|l|l|l|l|}
\hline Variable & Severity & Occurrence & Detection \\
\hline Very High & $\begin{array}{l}\text { Patient serious infection/personnel injury; device } \\
\text { scrapped/damaged severely }\end{array}$ & $\begin{array}{l}\text { Very high, failure is almost } \\
\text { inevitable }\end{array}$ & Almost impossible to be detected \\
\hline High & $\begin{array}{l}\text { Patient infection, cicatrisation delay; increase sur- } \\
\text { gery/in-hospital time/medical fees obviously; De- } \\
\text { vice damaged, serious failure }\end{array}$ & $\begin{array}{l}\text { High, failure is possible to } \\
\text { repeat }\end{array}$ & Difficult to be detected \\
\hline Moderate & $\begin{array}{l}\text { Effects on body, increase surgery time/expense; } \\
\text { device failure/slight damage }\end{array}$ & $\begin{array}{l}\text { Moderate, failure occurs occa- } \\
\text { sionally }\end{array}$ & Detected occasionally \\
\hline Low & $\begin{array}{l}\text { No effects on body safety, but related personnel } \\
\text { realize the device use not to be proper/smooth }\end{array}$ & Low, relatively few failures & Easy to be detected \\
\hline Very Low & No effects on device/personnel & Less likely to occur & Almost certain to be detected \\
\hline
\end{tabular}

To determine the triangle fuzzy numbers according to fuzzy language, the Delphi technique can be applied. Assume that there are $\mathrm{n}$ experts, each of whom has a weight of $\lambda_{i}(i=1,2,3, \ldots, n)$, and whose fuzzy evaluation of a certain language variable in failure modes is $\left(a_{i}, b_{i}, c_{i}\right)$. Then on the basis of experts' opinions, the integrated triangle fuzzy number corresponding to the fuzzy language of this variable could be obtained as Eq.s (Fout! Verwijzingsbron niet gevonden.) and (Fout! Verwijzingsbron niet gevonden.). In fuzzy theory, defuzzification of fuzzy numbers is the premise of applying grey relational theory, which could be achieved through Eq (5).

$$
\begin{array}{ll}
a=\sum_{i=1}^{n} \lambda_{i} a_{i} & \text { Equation (2) } \\
b=\sum_{i=1}^{n} \lambda_{i} b_{i} & \text { Equation (3) } \\
c=\sum_{i=1}^{n} \lambda_{i} c_{i} & \text { Equation (4) } \\
A(x)=\frac{(a+2 b+c)}{4} & \text { Equation (5) }
\end{array}
$$

In this example, the fuzzy numbers of three language variables $(\mathrm{S}, \mathrm{O}, \mathrm{D})$ is determined as is shown in Fout! Verwijzingsbron niet gevonden. and Fout! Verwijzingsbron niet gevonden. 


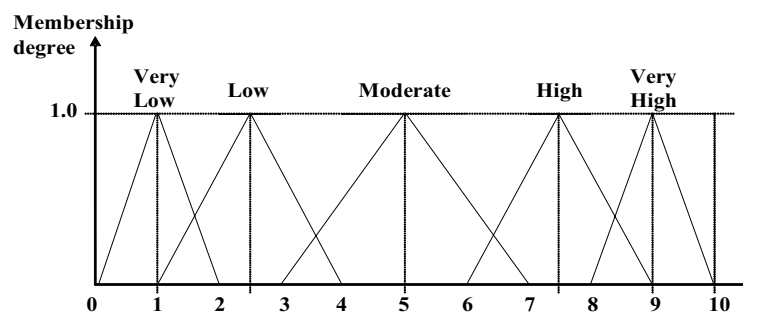

Figure 3 Membership function of language variables Table 3

Fuzzy numbers of language variables

\begin{tabular}{|l|l|}
\hline Variable & Fuzzy Number \\
\hline Very Low & $(0,1,2)$ \\
\hline Low & $(1,2.5,4)$ \\
\hline Moderate & $(3,5,7)$ \\
\hline High & $(6,7.5,9)$ \\
\hline Very High & $(8,9,10)$ \\
\hline
\end{tabular}

\subsubsection{FMEA team evaluation}

The FMEA team is composed of 5 experts related to the use of $\mathrm{C}$-arm machine. Their jobs and evaluation weights are: 1 attending physician (0.2), 2 device operator $(0.2$ each $), 1$ device maintenance man $(0.25)$ and 1 medical manager $(0.15)$.

Each member of the FMEA team needs to give assessment on $\mathrm{S}, \mathrm{O}$ and $\mathrm{D}$ of each failure mode, according to the fuzzy language variables and their fuzzy numbers. Through weight combination, as well as integration according to Formula 2, 3, 4, and defuzzification according to Formula 5, we get Table 4:

Table 4

Experts investigation in use-related risk for C-arm machine based on FMEA

\begin{tabular}{|c|c|c|c|c|c|c|c|}
\hline Failure mode & Severity & Severity* & $\begin{array}{l}\text { Failure } \\
\text { causes }\end{array}$ & Occurrence & Occurrence* & Detection & Detection* \\
\hline \multirow{2}{*}{$\mathrm{F}_{1}$} & \multirow{2}{*}{$(4.7,6.4,8.1)$} & \multirow{2}{*}{6.4} & $\mathrm{~F}_{11}$ & $(2.8,4.3,5.8)$ & 4.3 & $(2.5,4.4,6.3)$ & 4.4 \\
\hline & & & $\mathrm{F}_{12}$ & $(3 \cdot 0,5 \cdot 0,7.0)$ & 5.0 & $(2.7,4.6,6.6)$ & 4.6 \\
\hline \multirow{3}{*}{$\mathrm{F}_{2}$} & \multirow{3}{*}{$(4.4,5.8,7.2)$} & \multirow{3}{*}{5.8} & $\mathrm{~F}_{21}$ & $(2.5,3.9,5.3)$ & 3.9 & $(1.8,3.1,4.5)$ & 3.1 \\
\hline & & & $\mathrm{F}_{22}$ & $(2.5,3.9,5.3)$ & 3.9 & $(2.8,4.5,6.2)$ & 4.5 \\
\hline & & & $\mathrm{F}_{23}$ & $(2.4,3.7,5.4)$ & 3.8 & $(2.8,4.5,6.2)$ & 4.5 \\
\hline \multirow{2}{*}{$\mathrm{F}_{3}$} & \multirow{2}{*}{$(4.1,5.5,7.0)$} & \multirow{2}{*}{5.5} & $\mathrm{~F}_{31}$ & $(5 \cdot 0,6.0,7.1)$ & 6.0 & $(2.8,4.2,5.6)$ & 4.2 \\
\hline & & & $\mathrm{F}_{32}$ & $(5.3,6.7,8.2)$ & 6.7 & $(1.5,3.0,4.3)$ & 3.0 \\
\hline \multirow{3}{*}{$\mathrm{F}_{4}$} & \multirow{3}{*}{$(4.7,6.3,7.75)$} & \multirow{3}{*}{6.3} & $\mathrm{~F}_{41}$ & $(0.6,1.9,3.2)$ & 1.9 & $(0.4,1.9,3.2)$ & 1.9 \\
\hline & & & $\mathrm{F}_{42}$ & $(2.3,3.9,5.5)$ & 3.9 & $(1.6,3.2,4.8)$ & 3.2 \\
\hline & & & $\mathrm{F}_{43}$ & $(3.3,4.9,6.5)$ & 4.9 & $(2.6,3.9,5.2)$ & 3.9 \\
\hline \multirow{4}{*}{$\mathrm{F}_{5}$} & \multirow{4}{*}{$(2.5,4.4,6.3)$} & \multirow{4}{*}{4.4} & $\mathrm{~F}_{51}$ & $(2.3,3.9,5.5)$ & 3.9 & $(2.7,4.4,6.1)$ & 4.4 \\
\hline & & & $\mathrm{F}_{52}$ & $(2.0,3.5,5.0)$ & 3.5 & $(1.2,2.6,4.1)$ & 2.6 \\
\hline & & & $\mathrm{F}_{53}$ & $(3.0,4.6,6.1)$ & 4.6 & $(2.7,4.6,6.6)$ & 4.6 \\
\hline & & & $\mathrm{F}_{54}$ & $(3.3,4.8,6.3)$ & 4.8 & $(2.0,3.6,5.3)$ & 3.6 \\
\hline \multirow{3}{*}{$\mathrm{F}_{6}$} & \multirow{3}{*}{$(4.0,5.6,7.6)$} & \multirow{3}{*}{5.7} & $\mathrm{~F}_{61}$ & $(2.7,4.3,5.9)$ & 4.3 & $(3.8,5.1,6.5)$ & 5.1 \\
\hline & & & $\mathrm{F}_{62}$ & $(2.1,3.5,4.9)$ & 3.5 & $(1.8,3.1,4.5)$ & 3.1 \\
\hline & & & $\mathrm{F}_{63}$ & $(1.9,3.3,4.7)$ & 3.3 & $(2.9,4.5,6.2)$ & 4.5 \\
\hline \multirow{4}{*}{$\mathrm{F}_{7}$} & \multirow{4}{*}{$(5.2,6.8,8.4)$} & \multirow{4}{*}{6.8} & $\mathrm{~F}_{71}$ & $(3.8,5.1,6.5)$ & 5.1 & $(1.2,2.6,4.1)$ & 2.6 \\
\hline & & & $\mathrm{F}_{72}$ & $(5.3,6.4,7.55)$ & 6.4 & $(2.9,4.5,6.2)$ & 4.5 \\
\hline & & & $\mathrm{F}_{73}$ & $(2.7,4.4,6.1)$ & 4.4 & $(2.5,4.1,5.8)$ & 4.1 \\
\hline & & & $\mathrm{F}_{74}$ & $(3.3,4.7,6.1)$ & 4.7 & $(2.2,3.6,5.1)$ & 3.6 \\
\hline \multirow{2}{*}{$\mathrm{F}_{8}$} & \multirow{2}{*}{$(4.1,5.4,6.8)$} & \multirow{2}{*}{5.4} & $\mathrm{~F}_{81}$ & $(2.8,4.2,5.6)$ & 4.2 & $(0.7,2.0,3.3)$ & 2.0 \\
\hline & & & $\mathrm{F}_{82}$ & $(0.6,1.8,3.1)$ & 1.8 & $(1.4,2.8,4.3)$ & 2.8 \\
\hline
\end{tabular}

In the table, the columns filled with grey background are the defuzzified values for $\mathrm{S}, \mathrm{O}, \mathrm{D}$. 


\subsection{Integration of grey relational theory}

First of all, the defuzzified values are arranged as a comparison matrix $\left\{F_{i}(k)\right\}$. The sorting of failure modes can be carried out by building a reference matrix. The reference list of risk factors is determined by the ideal values of all the factors in FMEA, which can also be represented as a matrix. In this article, the worst value of all the variables is selected to build the reference matrix $\left\{F_{o}(k)\right\}$. According to grey relational theory, the coefficient between the variables in failure mode and reference could be calculated $\left(\gamma\left(F_{o}(k), F_{i}\right.\right.$ $(k)))$.

$$
\left\{F_{i}(k)\right\}=\left[\begin{array}{ccc}
6.4 & 4.3 & 4.4 \\
6.4 & 5.0 & 4.6 \\
5.8 & 3.9 & 3.1 \\
F_{1}(k) \\
\vdots .8 & 3.9 & 4.5 \\
5.8 & 3.8 & 4.5 \\
5.5 & 6.0 & 4.2 \\
F_{23}(k)
\end{array}\right]=\left[\begin{array}{ccc}
5.5 & 6.7 & 3.0 \\
6.3 & 1.9 & 1.9 \\
6.3 & 3.9 & 3.2 \\
6.3 & 4.9 & 3.9 \\
4.4 & 3.9 & 4.4 \\
4.4 & 3.5 & 2.6 \\
4.4 & 4.6 & 4.6 \\
4.4 & 4.8 & 3.6 \\
5.7 & 4.3 & 5.1 \\
5.7 & 3.5 & 3.1 \\
5.7 & 3.3 & 4.5 \\
6.8 & 5.1 & 2.6 \\
6.8 & 6.4 & 4.5 \\
6.8 & 4.4 & 4.1 \\
6.8 & 4.7 & 3.6 \\
5.4 & 4.2 & 2.0 \\
5.4 & 1.8 & 2.8
\end{array}\right]
$$$$
\left\{F_{0}(k)\right\}=\left[\begin{array}{ccc}
V H & V H & V H \\
\vdots & \vdots & \vdots \\
V H & V H & V H
\end{array}\right]=\left[\begin{array}{ccc}
10 & 10 & 10 \\
\vdots & \vdots & \vdots \\
10 & 10 & 10
\end{array}\right]
$$

$$
\begin{array}{r}
\gamma\left(F_{0}(k), F_{i}(k)\right) \\
=\frac{\min _{i} \min _{k}\left|F_{0}(k)-F_{i}(k)\right|+\xi \min _{i} \min _{k}\left|F_{0}(k)-F_{i}(k)\right|}{\left|F_{0}(k)-F_{i}(k)\right|+\xi \min _{i} \min _{k}\left|F_{0}(k)-F_{i}(k)\right|} \\
\text { Equation (8) }
\end{array}
$$

Here the identification coefficient $\xi$ is considered 0.5 . Thus the relational coefficient matrix is $\mathrm{R}$.

$$
R=\left[\begin{array}{ccc}
0.923 & 0.658 & 0.667 \\
0.923 & 0.727 & 0.686 \\
0.828 & 0.623 & 0.565 \\
0.828 & 0.623 & 0.676 \\
\gamma_{2}(k) \\
0.828 & 0.615 & 0.676 \\
0.787 & 0.857 & 0.649 \\
0.787 & 0.980 & 0.558 \\
0.906 & 0.495 & 0.495 \\
0.906 & 0.623 & 0.571 \\
0.906 & 0.716 & 0.623 \\
0.667 & 0.623 & 0.667 \\
0.667 & 0.593 & 0.533 \\
0.667 & 0.686 & 0.686 \\
0.667 & 0.706 & 0.600 \\
0.814 & 0.658 & 0.738 \\
0.814 & 0.593 & 0.565 \\
0.814 & 0.578 & 0.676 \\
1.000 & 0.738 & 0.533 \\
1.000 & 0.923 & 0.676 \\
1.000 & 0.667 & 0.640 \\
1.000 & 0.696 & 0.600 \\
0.774 & 0.649 & 0.500 \\
0.774 & 0.490 & 0.545
\end{array}\right]
$$

Equation (9)

Grey relational degree can be calculated through

$$
\Gamma\left(F_{0}, F_{i}\right)=\sum_{i=1}^{3} \lambda_{k} \times \gamma\left(F_{0}(k), F_{i}(k)\right)
$$

Equation (10)

So the failure mode grey relational degrees are listed in descending order as Fout! Verwijzingsbron niet gevonden. 
Table 5

$\mathrm{C}$-arm machine use-related failure mode ranking

\begin{tabular}{|l|l|l|l|l|l|}
\hline $\begin{array}{l}\text { Failure } \\
\text { mode }\end{array}$ & $\begin{array}{l}\text { Relational } \\
\text { coefficient }\end{array}$ & Order & $\begin{array}{l}\text { Failure } \\
\text { mode }\end{array}$ & $\begin{array}{l}\text { Relational } \\
\text { coefficient }\end{array}$ & Order \\
\hline $\mathrm{F}_{72}$ & 0.901 & 1 & $\mathrm{~F}_{23}$ & 0.702 & 13 \\
\hline $\mathrm{F}_{32}$ & 0.828 & 2 & $\mathrm{~F}_{21}$ & 0.683 & 14 \\
\hline $\mathrm{F}_{31}$ & 0.791 & 3 & $\mathrm{~F}_{63}$ & 0.680198 & 15 \\
\hline $\mathrm{F}_{71}$ & 0.789 & 4 & $\mathrm{~F}_{53}$ & 0.679048 & 16 \\
\hline $\mathrm{F}_{12}$ & 0.787 & 5 & $\mathrm{~F}_{54}$ & 0.67098 & 17 \\
\hline $\mathrm{F}_{74}$ & 0.783 & 6 & $\mathrm{~F}_{62}$ & 0.664354 & 18 \\
\hline $\mathrm{F}_{73}$ & 0.778 & 7 & $\mathrm{~F}_{81}$ & 0.66286 & 19 \\
\hline $\mathrm{F}_{43}$ & 0.764 & 8 & $\mathrm{~F}_{51}$ & 0.647186 & 20 \\
\hline $\mathrm{F}_{11}$ & 0.752 & 9 & $\mathrm{~F}_{41}$ & 0.638631 & 21 \\
\hline $\mathrm{F}_{61}$ & 0.728 & 10 & $\mathrm{~F}_{52}$ & 0.606667 & 22 \\
\hline $\mathrm{F}_{42}$ & 0.712 & 11 & $\mathrm{~F}_{82}$ & 0.600467 & 23 \\
\hline $\mathrm{F}_{22}$ & 0.705 & 12 & & & \\
\hline
\end{tabular}

\section{Conclusions}

From the above calculation, it's easy to identify the risk level of different use-related failure mode. A successful FMEA activity helps a team to identify potential failure modes, enabling the team to design those failures out of the system with the minimum of effort and resource expenditure, thereby reducing development time and costs. Besides, risk controls measures can also be taken in operations management, which means implementing use-related risk control for medical devices from the perspectives of people, devices, environment, system, etc., and from the levels of device use, human factor control, management specification, etc.

\section{References}

[1] Binhua Yuan, Reflections on risk prevention and supervision of unsafe medical equipments, China International Medical Equipment Application Security and Quality Management Forum, 2007.

[2] Health Grades, Inc. Patient safety in American hospitals, 2004

[3] Robin E., McDermott, Raymond J., et al, The basics of FMEA, Portland, OR: Resources Engineering, 1996.

[4] Langford J.W., Logistics: Principles and applications, McGraw Hill, 1995.

[5] Chiozza M.L., Ponzetti C., FMEA: a model for reducing medical errors, Clinica Chimica Acta (40), 2009: 75-78.

[6] Xu Ping, Xu Min, Xing Maoying, et al, The application and limitation of FMEA in medical risk management, Modern Preventive Medicine, 2007, 34(1): 51-52.
[7] Lunbiao Yang, Yingyi Gao, Fuzzy mathematics theory and application, Guangzhou: South China University Technology Press, 1993. 